\title{
American Society of Anesthesiologists physical status score as a predictor of long-term outcome in women with endometrial cancer
}

\section{Kolehmainen, Anne Maarit}

2019-06

Kolehmainen , A M , Pasanen , A , Tuomi , T , Koivisto-Korander , R , Butzow , R \& Loukovaara, M 2019 , ' American Society of Anesthesiologists physical status score as a predictor of long-term outcome in women with endometrial cancer ' , International Journal of Gynecological Cancer , vol. 29 , no. 5 , pp. 879-885 . https://doi.org/10.1136/ijgc-2018-000118

http://hdl.handle.net/10138/311370

https://doi.org/10.1136/ijgc-2018-000118

cc_by_nc

acceptedVersion

Downloaded from Helda, University of Helsinki institutional repository.

This is an electronic reprint of the original article.

This reprint may differ from the original in pagination and typographic detail.

Please cite the original version. 


\section{The American Society of Anesthesiologists physical status score as a predictor of long-term outcome in women with endometrial cancer}

Anne Kolehmainen, MD,* Annukka Pasanen, MD, $†$ Taru Tuomi, MD,* Riitta Koivisto-Korander, MD,* Ralf Bützow, MD, $\dagger$ Mikko Loukovaara, MD*

*Department of Obstetrics and Gynecology, Helsinki University Hospital and University of Helsinki, Helsinki, Finland (anne.a.kolehmainen@hus.fi, taru.tuomi@hus.fi, riitta.koivisto-korander@hus.fi, mikko.loukovaara@hus.fi)

$†$ Department of Pathology, Helsinki University Hospital and Research Program in Applied Tumor Genomics, Faculty of Medicine, University of Helsinki, Helsinki, Finland (annukka.pasanen@ @us.fi, ralf.butzow@hus.fi)

Corresponding author: Anne Kolehmainen, MD

Department of Obstetrics and Gynecology, Helsinki University Hospital and University of Helsinki, P.O. Box 140, 00029 HUS, Helsinki, Finland. Tel: +358504286988. Fax: +358947173640. E-mail address: anne.a.kolehmainen@hus.fi (A. Kolehmainen)

Acknowledgments: None

Disclosure statement: The authors declare no conflicts of interest.

Funding: This study was supported by Helsinki University Hospital research funds. 


\section{Abstract}

Objectives: To study the association of the American Society of Anesthesiologists (ASA) physical status score with long-term outcome in endometrial cancer.

Methods: Overall, disease-specific and non-cancer related survival were estimated using simple and multivariable Cox regression analyses and the Kaplan-Meier method.

Results: A total of 1166 patients were included in the study. Median follow-up time was 76 months (range 1-136). All-cause and non-cancer related mortality were increased in patients whose ASA physical status score was III (hazard ratios 2.5 and 8.0, respectively) or IV (hazard ratios 5.7 and 25, respectively), and cancer-related mortality was increased in patients whose score was IV (hazard ratio 2.7). Kaplan-Meier analyses demonstrated a worse overall, disease-specific and non-cancer related survival for patients whose score was $\geq$ III $(\mathrm{P}<0.0001$ for all). Disease-specific survival was also separately analyzed for patients with stage I and stage II-IV cancer. Compared with patients whose score was $\leq$ II, the survival was worse for patients whose score was $\geq \mathrm{III}$ in both subgroups of stages $(\mathrm{P}=0.003$ and $\mathrm{P}=0.017$ for stage I and stages II-IV, respectively). ASA physical status score remained an independent predictor of all-cause mortality (hazard ratio 2.2 for scores $\geq \mathrm{III}$ ), cancer-related mortality (hazard ratios 1.7 and 2.2 for scores $\geq$ III and IV, respectively) and non-cancer related mortality (hazard ratio 3.1 for scores $\geq$ III) after adjustment for prognostically relevant clinicopathologic and blood-based covariates. ASA physical status score also remained an independent predictor of cancer-related mortality after exclusion of patients who were at risk for nodal involvement based on features of the primary tumor but did not undergo lymphadenectomy, and patients with an advanced disease who received suboptimal chemotherapy (hazard ratios 1.6 and 2.5 for scores $\geq \mathrm{III}$ and IV, respectively).

Conclusions: ASA physical status score independently predicts overall survival, disease-specific survival and non-cancer related survival in endometrial cancer.

Key Words: American Society of Anesthesiologists, Endometrial cancer, Physical status score 


\section{Introduction}

Endometrial cancer is the sixth most common cancer in women worldwide, with highest rates in North America and Eastern and Northern Europe [1]. It is diagnosed predominantly in elderly women, the median age being 62 years at diagnosis [2]. Compared with the general population, women with endometrial cancer have an increased prevalence of comorbid conditions, most importantly obesity and diabetes [3].

The American Society of Anesthesiologists (ASA) physical status classification system was developed in 1941 to offer clinicians a simple categorization of a patient's physiological status that can be helpful in predicting operative risk [4]. The time frame around the perioperative period that the ASA physical status classification system encompasses has not been defined. The latest version of the classification system was approved in 2014 [5]. This update included examples of patient characteristics that fit into each ASA physical status score, scaled from I to VI (Supplemental Table 1).

Although the ASA physical status classification system is mainly used for prediction of perioperative morbidity and mortality, there is some evidence that it may also predict long-term outcome of cancer patients. In those undergoing radical surgery for urinary bladder cancer or upper tract urothelial carcinoma, ASA physical status score independently predicts overall mortality [6-8]. Here, we wanted to test a hypothesis that high ASA physical status score is associated with an unfavorable long-term outcome in women with endometrial cancer.

\section{Materials and Methods}

Patients who underwent primary surgical treatment for endometrial cancer at the Department of Obstetrics and Gynecology, Helsinki University Hospital, between January 1, 2007 and December 31, 2013 were included in this study. The study was approved by the Institutional Review Board (journal number 135/13/03/03/2013) and the National Supervisory Authority for Welfare and Health (journal number 753/06.01.03.01/2016). Clinicopathologic data were abstracted from institutional medical and pathology 
records. ASA physical status scores were abstracted from anesthesia records and then revised to comply with the 2014 update of the ASA physical status classification system [5]. Specifically, current smokers and subjects with obesity $\left(30 \mathrm{~kg} / \mathrm{m}^{2}<\right.$ body mass index $\left.<40 \mathrm{~kg} / \mathrm{m}^{2}\right)$ or well-controlled diabetes were classified as ASA physical status score II patients, whereas those with severe obesity (body mass index $\geq 40 \mathrm{~kg} / \mathrm{m}^{2}$ ) were classified as ASA physical status score III patients. Body mass index was unknown for one patient whose ASA physical status score was IV. Stage was determined according to the International Federation of Gynecology and Obstetrics guidelines revised in 2009 [9].

Follow-up data were last updated in January-March 2018, together with clinicopathologic data where appropriate. All survival times were calculated from the date of surgery to death to obviate the measurement error due to imprecision in estimating the day of diagnosis. Cause of death was mainly based on medical records. Missing data were complemented from death certificates derived from Statistics Finland.

The standard surgical treatment was total hysterectomy with bilateral salpingooophorectomy. Lymphadenectomy was performed in selected patients. Adjuvant therapy was tailored according to stage and histologic findings at surgery. Patients with early stage endometrioid cancer with high risk features generally received either vaginal brachytherapy or whole pelvic radiotherapy, and patients with nonendometrioid or advanced (stage III-IV) endometrioid cancer received multimodality treatment with chemotherapy and radiation.

Variables of the last pretreatment complete blood count were analyzed by photometric measurement (hemoglobin) and electrical impedance technology and flow cytometry (cells). Anemia was defined as blood hemoglobin concentration $<117 \mathrm{~g} / \mathrm{L}$, leukocytosis as a leukocyte count $>8.2 \times 10^{9} / \mathrm{L}$, and thrombocytosis as a platelet count $>360 \times 10^{9} / \mathrm{L}$. These values represent the reference intervals for Finnish adult females [10]. Blood count variables were not available for one patient. Pretreatment CA125 concentration was quantitated with a chemiluminescent microparticle immunoassay on the Abbott 
Architect 2000i Analyzer (Abbott Diagnostics, Abbott Park, Illinois, USA). The concentration was considered increased when $>35 \mathrm{U} / \mathrm{mL}$ [11]. The value of CA125 was not available for 121 patients.

Pearson $\chi^{2}$ or Fisher exact test was used for comparisons of categorical variables. Survivals were estimated using simple and multivariable Cox regression analyses and the Kaplan-Meier method. Differences between groups were compared using the log rank test. Statistical significance was set at $\mathrm{P}<$ 0.05. Data were analyzed using the Statistical Package for the Social Sciences version 24 software (IBM Corp., Armonk, New York, USA).

\section{Results}

A total of 1166 patients were included in the study (Table 1). Of these, the ASA physical status score was I in $49(4.2 \%)$, II in $478(41.0 \%)$, III in $548(47.0 \%)$, and IV in $91(7.8 \%)$. None of the patients had a score of V or VI.

Of the patients who had stage IA-IIIC2 endometrioid cancer and were at low-intermediate to high risk for lymph node involvement according to the Milwaukee criteria of primary tumors [12], $69.2 \%$ (323/467) underwent pelvic or pelvic-aortic lymphadenectomy. Of those at low risk [12], 57.9\% (287/496) underwent lymphadenectomy (due to missing data, denominators do not add up to 1027, which was the total number of stage IA-IIIC2 endometrioid cancers). Of the patients with stage IA-IIIC2 nonendometrioid cancer, 79.4\% (77/97) underwent lymphadenectomy. Two patients with stage IVB cancer underwent surgical exploration without hysterectomy.

Of the 203 patients who were treated with at least one cycle of combination chemotherapy, $164(80.8 \%)$ received paclitaxel and carboplatin and $36(17.7 \%)$ received epirubicin and carboplatin. Paclitaxel was changed to docetaxel in 17 patients because of paclitaxel toxicity, and two (1.0\%) patients received docetaxel as a taxane drug in all treatment cycles. One patient $(0.5 \%)$ received first epirubicin and then paclitaxel in combination with carboplatin. Forty-four patients received carboplatin monotherapy. 
Seven patients died within 30 days after surgery, which gives a rate of $0.6 \%$ for perioperative mortality. Of these seven patients, the ASA physical status score was II in one, III in three, and IV in three. Six patients had stage IVB cancer. One patient whose ASA physical status score was IV had stage IA cancer, and she was the only patient whose perioperative death was not caused by endometrial cancer. Of the $304(26.1 \%)$ patients who died during follow-up, $176(15.1 \%)$ died from endometrial cancer and 128 (11.0\%) from other causes. Median follow-up time was 76 months (range 1136).

Simple Cox regression analyses were performed with ASA physical status score I as reference (Table 2). All-cause mortality and non-cancer related mortality were increased in patients whose score was III or IV, and cancer-related mortality was increased in patients whose score was IV. Risk of death was not altered in patients whose score was II.

Covariates for the multivariable survival analyses were selected based on unadjusted analyses of potential risk factors (Table 2). In addition to the ASA physical status score, the following covariates showed significant associations with all-cause mortality and were included in the multivariable analysis: age, diabetes, histology, stage, blood hemoglobin concentration and leukocyte count, serum CA125 concentration, and type of adjuvant therapy. In the multivariable analysis, ASA physical status score $\geq$ III, age $\geq 60$, endometrioid grade 3 and nonendometrioid histology, stage II-IV, anemia and elevated serum CA125 increased all-cause mortality (Table 3). Whole pelvic radiotherapy, chemotherapy and multimodality treatment decreased all-cause mortality (Table 3).

Diabetes was not significantly associated with cancer-related mortality in unadjusted analyses. It was excluded from the multivariable analysis of cancer-related mortality, which demonstrated ASA physical status score, age, histology, stage, serum CA125 and type of adjuvant therapy as independent prognosticators (Table 3). We also re-ran the same model with the cut-off for ASA physical status score set at III instead of IV. ASA physical status score remained an independent predictor of cancer-related mortality (hazard ratio $1.7,95 \%$ confidence interval 1.2-2.4; $\mathrm{P}=0.002$ ). 
Of the patients with stage I-IIIC2 endometrioid cancer who did not satisfy the low-risk Milwaukee criteria for lymph node involvement [12], the lymphadenectomy rate was $84.3 \%(167 / 198)$ in those whose ASA physical status score was I or II, as opposed to $58.0 \%$ (156/269) in those whose score was III or IV (P < 0.0001). In patients with stage I-IIIC2 nonendometrioid cancer, the lymphadenectomy rate was $87.9 \%$ (29/33) in those whose ASA physical status score I or II, and $75.0 \%(48 / 64)$ in those whose score was higher $(\mathrm{P}=0.188)$. In the subgroup of patients with stage III-IV cancer, $78.5 \%(62 / 79)$ of those with an ASA physical status score of I or II and 55.2\% (64/116) of those with a score of III or IV received chemotherapy with a curative intent, defined as a minimum of six cycles of combination chemotherapy $(\mathrm{P}=0.001)$. When patients who were allowed to forgo lymphadenectomy despite not satisfying the low-risk Milwaukee criteria [12] and patients with stage III-IV cancer who received suboptimal chemotherapy were excluded from the multivariable analysis of cancer-related mortality, ASA physical status score remained an independent predictor of poor outcome at a cut-off of III (hazard ratio $1.6,95 \%$ confidence interval $1.1-2.3 ; \mathrm{P}=0.011$ ) and $\mathrm{IV}$ (hazard ratio $2.5,95 \%$ confidence interval 1.4-4.4; $\mathrm{P}=0.001)$.

ASA physical status score, age, diabetes, histology, anemia and CA125 were associated with non-cancer related mortality in unadjusted analyses (Table 2). ASA physical status score and age were the strongest predictors of non-cancer related mortality in multivariable analysis (Table 3).

Of the 217 diabetic patients, 193 (88.9\%) had an ASA physical status score of III or IV due to poor control of diabetes or additional comorbidities. Because of the obvious overlap between ASA physical status score and diabetes as covariates in multivariable analyses of all-cause mortality and noncancer related mortality (Table 3), these analyses were also performed after excluding either ASA physical status score or diabetes. Hazard ratios remained essentially unaltered for the remaining covariates; however, after exclusion of ASA physical status score, diabetes showed a significant effect on both all-cause mortality (hazard ratio $1.4,95 \%$ confidence interval 1.1-1.9; $\mathrm{P}=0.018$ ) and non-cancer related mortality (hazard ratio $2.1,95 \%$ confidence interval $1.4-3.1 ; \mathrm{P}=0.001$ ). 
Based on multivariable analyses, ASA physical status score III was chosen as the cut-off for Kaplan-Meier survival analyses. These plots demonstrated a worse overall survival, disease-specific survival and non-cancer related survival for patients whose ASA physical status score was $\geq$ III (Figure 1A-C). Disease-specific survival was also separately analyzed for patients with stage I and stage II-IV cancer. Compared with patients whose ASA physical status score was $\leq \mathrm{II}$, the survival was worse for patients whose score was $\geq$ III in both subgroups of stages (Figure 2A-B).

\section{Discussion}

Prognostic factors for endometrial cancer have been extensively investigated, with an intention to provide tools for individualized treatment, tailored follow-up, and patient counseling. Stage and features of the primary tumor, such as histologic subtype, grade, depth of myometrial invasion and lymphovascular space invasion, are among the most commonly used prognosticators in clinical practice [13]. Many tissue biomarkers, e.g. L1CAM [14,15] and estrogen and progesterone receptor status [16], have been proposed as molecular determinants of patient outcome. Of the blood-based assays, CA125 [17] and HE4 [18] have been suggested to serve as prognostic biomarkers in endometrial cancer. Moreover, abnormalities in the preoperative complete blood count, i.e. anemia, leukocytosis and thrombocytosis, appear to be poor prognostic findings [19]. However, none of the tissue biomarkers or blood-based tests are widely used in gynecologic oncology practices. The Cancer Genome Atlas molecular classification, which divides endometrial cancers into four distinct categories [20], improves assessment of prognosis compared with conventional risk factors alone and holds promise in reducing overtreatment and undertreatment $[21,22]$.

Of the clinical patient characteristics, high age has been found to be associated with poor outcome in endometrial cancer $[23,24]$. Specifically, age $\geq 60$ years is an independent predictor of locoregional relapses and disease-related death in stage I endometrial cancer [25], and was chosen as the cut-off for high age in the current study. Findings on the prognostic significance of obesity and diabetes are less consistent, but prevailing data suggest that they are associated with poor overall survival $[3,26]$. 
Data presented herein provide a novel prognostic instrument in women with endometrial cancer, as the ASA physical status score was found to be associated with poor long-term outcome. It could be argued that surgical understaging and/or weaker tolerance may have lead to a more frequent use of suboptimal adjuvant therapy in patients with a high ASA physical status score, and, consequently, worse outcome. The evidence for improved survival by adjuvant therapy in endometrial cancer is best for chemotherapy in advanced cases [13]. ASA physical status score remained an independent predictor of cancer-related mortality even after exclusion of patients who were at risk for nodal metastasis as per the Milwaukee criteria [12] but did not undergo lymphadenectomy, and patients with an advanced disease who received suboptimal chemotherapy. ASA physical status score also remained an independent predictor of cancer-related mortality after adjusting for the type of adjuvant therapy. Thus, differences in surgical treatment or adjuvant therapies unlikely explain our findings.

Hazard ratios were quite similar for all significant risk factors in the multivariable analyses of all-cause mortality and non-cancer related mortality. By contrast, compared with ASA physical status score, the hazard ratio was somewhat higher for stage in the multivariable analysis of cancer-related mortality. It should be remembered, however, that the hazard ratio for ASA physical status score did not differ from that for age, a risk factor that was strong enough to be included in two prognostic nomograms for endometrial cancer $[27,28]$. The ASA physical status classification system may similarly deserve attention in the prognostication of endometrial cancer. It is noteworthy that this classification system predicted disease-specific survival also in the subgroup of stage I cancers that sometimes tend to leave clinicians with uncertainty regarding the true potential for the disease to recur.

Despite the fact that the ASA physical status classification system was originally developed to predict operative risk [4], it seems unlikely that a high perioperative mortality can explain the worse outcome of patients with high ASA physical status scores during the whole follow-up time. Six of the seven patients who died within 30 days after surgery had an ASA physical status score of III or IV; all of them succumbed to stage IVB endometrial cancer, which generally has a very poor prognosis. 
We assume that the effect of obesity on the poor outcome of patients with a high ASA physical status score was not considerable because body mass index was not associated with increased all-cause, cancer-related or non-cancer related mortality in univariable analyses. Similarly, based on univariable analysis, diabetes was not associated with cancer-related mortality. The effect of diabetes may be more important on deaths unrelated to endometrial cancer because it increased all-cause mortality and non-cancer related mortality in multivariable analyses after exclusion of ASA physical status score as a covariate.

Our study is strengthened by the large sample size and prospectively maintained database with long follow-up time. One of the end-points was disease-specific survival, which is the ideal outcome of interest after a cancer diagnosis. Detailed clinicopathologic data allowed us to control for the most common confounding factors. Presumably, this mitigated the shortcomings associated with the retrospective design of the study.

Although the external validity of the current findings will need to be verified in other patient samples, it is reassuring that the stage distribution and proportion of nonendometrioid cancers were comparable to findings in the Gynecologic Oncology Group 210 surgical pathological staging study of 5866 patients, with the vast majority being local endometrioid cancers [29]. Further, the proportion of women who had at most a mild systemic disease, based on their ASA physical status score of I or II, was very similar to a population-based analysis of 5408 women who underwent hysterectomy for endometrial cancer across the United States (45\% and $43 \%$ in our study and in the population-based analysis, respectively) [30].

The biological mechanisms by which the ASA physical status score might have an impact on endometrial cancer-related mortality remain to be elucidated. However, the current findings can be considered to be consistent with an earlier report in which frailty, i.e. state of low functional reserve seen particularly in old subjects, predicted shortened overall survival and disease-free survival in women with endometrial cancer [31]. It could be speculated that the partly subjective nature of the ASA physical status 
classification system allows frailty and other difficultly measurable clinical findings to be translated into a worse classification [32]. Clearly, more knowledge is needed to better understand the interaction between physical fitness and cancer survival.

\section{References}

[1] Lortet-Tieulent J, Ferlay J, Bray F, et al. International patterns and trends in endometrial cancer incidence, 1978-2013. JNCI J Natl Cancer Inst. 2018;110:354-361.

[2] American Cancer Society. Endometrial cancer (Internet). Available at https://www.cancer.org/cancer/endometrial-cancer.html (accessed 23 November 2018).

[3] Ko EM, Walter P, Clark L, et al. The complex triad of obesity, diabetes and race in type I and II endometrial cancers: prevalence and prognostic significance. Obstet Gynecol. 2014;133:28-32.

[4] Saklad M. Grading of patients for surgical procedures. Anesthesiology 1941;2:281-284.

[5] American Society of Anesthesiologists. ASA physical status classification system (Internet). Available at https://www.asahq.org/resources/clinical-information/asa-physical-statusclassification-system (accessed 23 November 2018).

[6] Boorjian SA, Kim SP, Tollefson MK, et al. Comparative performance of comorbidity indices for estimating perioperative and 5-year all cause mortality following radical cystectomy for bladder cancer. J Urol. 2013;190:55-60.

[7] Djaladat H, Bruins HM, Miranda G, et al. The association of preoperative serum albumin level and American Society of Anesthesiologists (ASA) score on early complications and survival of patients undergoing radical cystectomy for urothelial bladder cancer. BJU Int. 2014;113:887-893.

[8] Kang HW, Seo SP, Kim WT, et al. Impact of the ASA physical status score on adjuvant chemotherapy eligibility and survival of upper tract urothelial carcinoma patients: a multicenter study. J Korean Med Sci. 2017;32:335-342. 
[9] Pecorelli S. Revised FIGO staging for carcinoma of the vulva, cervix, and endometrium. Int J Gynaecol Obstet. 2009;105:103-104.

[10] Kairisto V, Grönroos P, Loikkanen M, et al. New Finnish reference limits for the basic blood count. Suomen Lääkärilehti 2003;58:5147-5153.

[11] Bast RC Jr, Feeney M, Lazarus H, et al. Reactivity of a monoclonal antibody with human ovarian carcinoma. J Clin Invest. 1981;68:1331-1337.

[12] Cox Bauer CM, Greer DM, Kram JJF, et al. Tumor diameter as a predictor of lymphatic dissemination in endometrioid endometrial cancer. Gynecol Oncol. 2016;141:199-205.

[13] SGO Clinical Practice Endometrial Cancer Working Group, Burke WM, Orr J, Leitao M, et al. Endometrial cancer: a review and current management strategies: part II. Gynecol Oncol. 2014;134:393-402.

[14] Dellinger TH, Smith DD, Ouyang C, et al. L1CAM is an independent predictor of poor survival in endometrial cancer - an analysis of The Cancer Genome Atlas (TCGA). Gynecol Oncol. 2016;141:336-340.

[15] Pasanen A, Tuomi T, Isola J, et al. L1 cell adhesion molecule as a predictor of disease-specific survival and patterns of relapse in endometrial cancer. Int J Gynecol Cancer. 2016;26:1465-1471.

[16] Smith D, Stewart CJR, Clarke EM, et al. ER and PR expression and survival after endometrial cancer. Gynecol Oncol. 2018;148:258-266.

[17] Kim HS, Park CY, Lee JM, et al. Evaluation of serum CA-125 levels for preoperative counseling in endometrioid endometrial cancer: a multi-center study. Gynecol Oncol. 2010;118:283-288.

[18] Brennan DJ, Hackethal A, Metcalf AM, et al. Serum HE4 as a prognostic marker in endometrial cancer - a population based study. Gynecol Oncol. 2014;132:159-165.

[19] Njølstad TS, Engerud H, Werner HMJ, et al. Preoperative anemia, leukocytosis and thrombocytosis identify aggressive endometrial carcinomas. Gynecol Oncol. 2013;131:410-415. 
[20] The Cancer Genome Atlas Research Network, Kandoth C, Schultz N, Cherniack AD, et al. Integrated genomic characterization of endometrial carcinoma. Nature 2013;497:67-73.

[21] Stelloo E, Nout RA, Osse EM, et al. Improved risk assessment by integrating molecular and clinicopathological factors in early-stage endometrial cancer - combined analysis of the PORTEC cohorts. Clin Cancer Res. 2016;22:4215-4224.

[22] Talhouk A, McConechy MK, Leung S, et al. Confirmation of ProMisE: a simple, genomics-based clinical classifier for endometrial cancer. Cancer 2017;123:802-813.

[23] Vance S, Yechieli R, Cogan C, et al. The prognostic significance of age in surgically staged patients with type II endometrial carcinoma. Gynecol Oncol. 2012;126:16-19.

[24] Benedetti Panici P, Basile S, Salerno MG, et al. Secondary analyses from a randomized clinical trial: age as the key prognostic factor in endometrial carcinoma. Am J Obstet Gynecol. 2014;210:363.e1-10.

[25] Creutzberg CL, Nout RA, Lybeert MLM, et al. Fifteen-year radiotherapy outcomes of the randomized PORTEC-1 trial for endometrial carcinoma. Int J Radiation Oncology Biol Phys. 2011;81:e631-638.

[26] Secord AA, Hasselblad V, Von Gruenigen VE, et al. Body mass index and mortality in endometrial cancer: a systematic review and meta-analysis. Gynecol Oncol. 2016;140:184-190.

[27] Abu-Rustum NR, Zhou Q, Gomez JD, et al. A nomogram for predicting overall survival of women with endometrial cancer following primary therapy: toward improving individualized cancer care. Gynecol Oncol. 2010;116:399-403.

[28] Kondalsamy-Chennakesavan S, Yu C, Kattan MW, et al. Nomograms to predict isolated locoregional or distant recurrence among women with uterine cancer. Gynecol Oncol. 2012;125:520-525. 
[29] Creasman WT, Ali S, Mutch DG, et al. Surgical-pathological findings in type 1 and 2 endometrial cancer: an NRG Oncology/Gynecologic Oncology Group study on GOG-210 protocol. Gynecol Oncol. 2017;145:519-525.

[30] Wright JD, Chen L, Gabor L, et al. Patterns of specialty-based referral and perioperative outcomes for women with endometrial cancer undergoing hysterectomy. Obstet Gynecol. 2017;130:81-90.

[31] Driver JA, Viswanathan AN. Frailty measure is more predictive of outcomes after curative therapy for endometrial cancer than traditional risk factors in women 60 and older. Gynecol Oncol. 2017;145:526-530.

[32] Araujo BLC, Theobald D. Letter to the Editor: ASA physical status classification in surgical oncology and the importance of improving inter-rater reliability. $J$ Korean Med Sci. 2017;32:1211-1212.

\section{Figure legends}

Figure 1. Kaplan-Meier survival analyses concerning the American Society of Anesthesiologists (ASA) physical status classification system in patients with endometrial cancer.

Figure 2. Kaplan-Meier disease-specific survival analyses concerning the American Society of Anesthesiologists (ASA) physical status classification system in patients with stage I (A) and stage II-IV (B) endometrial cancer. 
Table 1. Clinicopathologic data $(n=1166)$.

\begin{tabular}{|c|c|}
\hline Clinicopathologic feature & Result \\
\hline Age (years) [median (interquartile range)] & $68(60-75)$ \\
\hline Body mass index $\left(\mathrm{kg} / \mathrm{m}^{2}\right)$ [median (interquartile range)]* & $27.5(24.0-32.4)$ \\
\hline Diabetes (number of cases, percent) $\dagger$ & $217(18.6 \%)$ \\
\hline Current smokers (number of cases, percent) & $125(10.7 \%)$ \\
\hline Minimally invasive hysterectomy (number of cases, percent) $\ddagger$ & $912(78.2 \%)$ \\
\hline Pelvic lymphadenectomy (number of cases, percent) & $580(49.7 \%)$ \\
\hline Pelvic-aortic lymphadenectomy (number of cases, percent) & $175(15.0 \%)$ \\
\hline $\begin{array}{l}\text { Histology (number of cases, percent) } \\
\text { Endometrioid carcinoma } \\
\text { Clear cell carcinoma } \\
\text { Serous carcinoma } \\
\text { Carcinosarcoma } \\
\text { Undifferentiated carcinoma } \\
\text { Neuroendocrine carcinoma }\end{array}$ & $\begin{array}{l}1041(89.3 \%) \\
43(3.7 \%) \\
43(3.7 \%) \\
20(1.7 \%) \\
18(1.5 \%) \\
1(0.1 \%) \\
\end{array}$ \\
\hline $\begin{array}{l}\text { Grade (number of cases, percent) (For endometrioid only, } \mathrm{n}=1041 \text { ) } \\
1 \\
2 \\
3\end{array}$ & $\begin{array}{l}643(55.1 \%) \\
265(22.7 \%) \\
133(11.4 \%) \\
\end{array}$ \\
\hline $\begin{array}{l}\text { Stage (number of cases, percent) } \\
\text { IA } \\
\text { IB } \\
\text { II } \\
\text { IIIA } \\
\text { IIIB } \\
\text { IIIC1 } \\
\text { IIIC2 } \\
\text { IVA } \\
\text { IVB }\end{array}$ & $\begin{array}{l}681(58.4 \%) \\
224(19.2 \%) \\
66(5.7 \%) \\
54(4.6 \%) \\
11(0.9 \%) \\
55(4.7 \%) \\
33(2.8 \%) \\
0(0 \%) \\
42(3.6 \%)\end{array}$ \\
\hline $\begin{array}{l}\text { Adjuvant therapy (number of cases, percent) } \\
\text { Vaginal brachytherapy } \\
\text { Pelvic radiotherapy } \\
\text { Chemotherapy } \\
\text { Chemotherapy and vaginal brachytherapy } \\
\text { Chemotherapy and pelvic radiotherapy }\end{array}$ & $\begin{array}{l}503(43.1 \%) \\
149(12.8 \%) \\
47(4.0 \%) \\
67(5.7 \%) \\
133(11.4 \%)\end{array}$ \\
\hline
\end{tabular}

*Number of cases 1165 (body mass index missing for one patient)

$\dagger$ Type 2 diabetes, $\mathrm{n}=215$; type 1 diabetes, $\mathrm{n}=2$

†Laparoscopic, $\mathrm{n}=829$ (including 51 conversions); robotic, $\mathrm{n}=72$; vaginal, $\mathrm{n}=11$ 
Table 2. Simple Cox regression analyses of all-cause mortality, cancer-related mortality and non-cancer related mortality $(\mathrm{n}=1166)$.

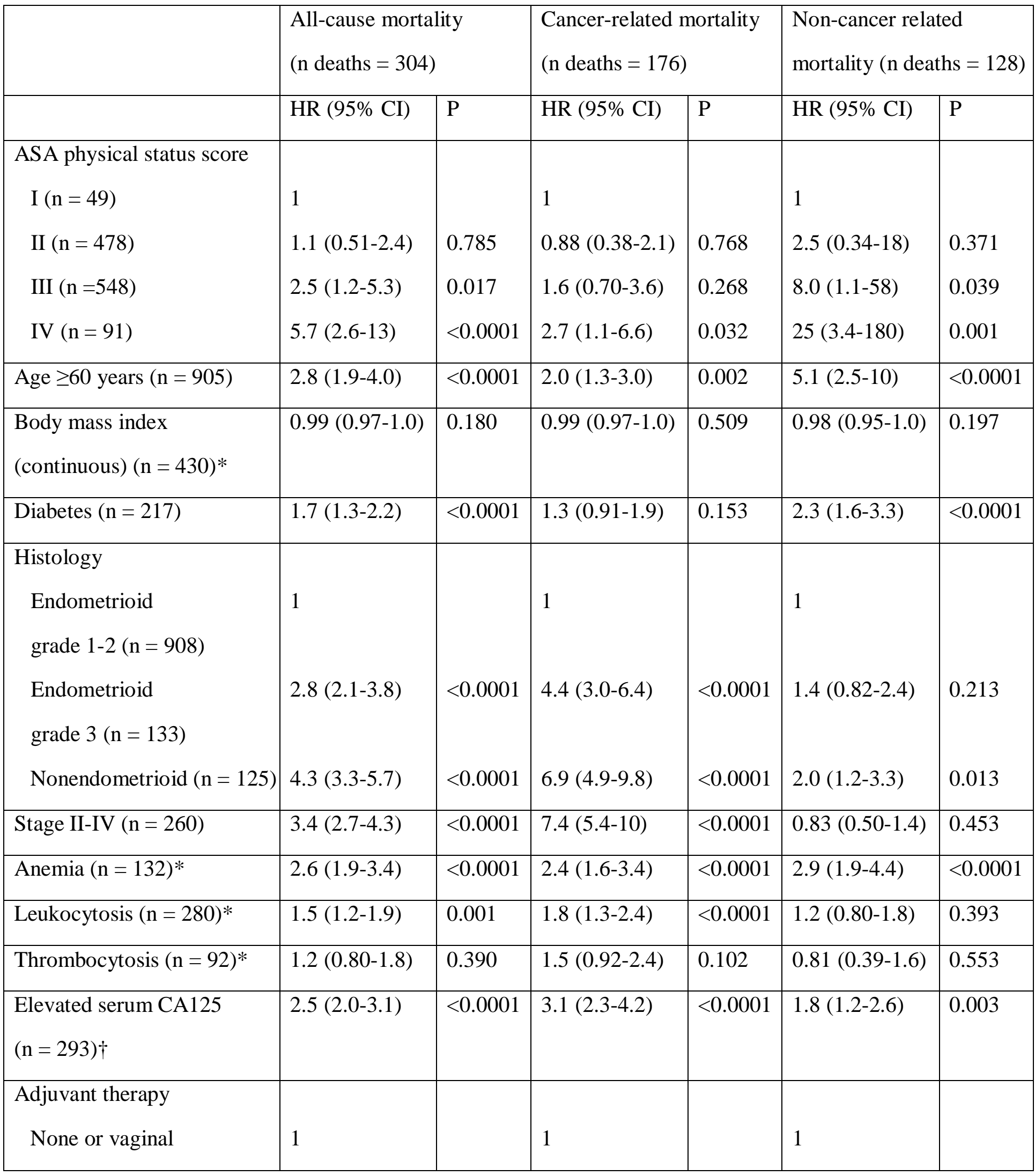




\begin{tabular}{|l|l|l|l|l|l|l|}
\hline $\begin{array}{l}\text { brachytherapy }(\mathrm{n}=770) \\
\text { Pelvic radiotherapy }\end{array}$ & $1.5(1.1-2.1)$ & 0.020 & $2.7(1.7-4.3)$ & $<0.0001$ & $0.74(0.40-1.3)$ & 0.315 \\
$(\mathrm{n}=149)$ & $3.2(2.3-4.3)$ & $<0.0001$ & $6.0(4.0-9.0)$ & $<0.0001$ & $1.3(0.73-2.3)$ & 0.361 \\
$\begin{array}{l}\text { Chemotherapy }(\mathrm{n}=114) \\
\begin{array}{l}\text { Chemotherapy and pelvic } \\
\text { radiotherapy }(\mathrm{n}=133)\end{array}\end{array}$ & $2.5(1.9-3.5)$ & $<0.0001$ & $5.5(3.7-8.0)$ & $<0.0001$ & $0.63(0.31-1.3)$ & 0.212 \\
\hline
\end{tabular}

*Data missing for one patient.

$\dagger$ Data missing for 121 patients.

ASA, American Society of Anesthesiologists

Table 3. Multivariable Cox regression analyses of all-cause mortality, cancer-related mortality and noncancer related mortality. Patients with available data for all of the selected covariates were included in the models $(n=1044)$.

\begin{tabular}{|l|l|l|l|l|l|l|}
\hline & \multicolumn{2}{|l|}{$\begin{array}{l}\text { All-cause mortality } \\
\text { (n deaths = 277) }\end{array}$} & \multicolumn{2}{l|}{$\begin{array}{l}\text { Cancer-related mortality } \\
\text { (n deaths = 164) }\end{array}$} & \multicolumn{2}{l|}{$\begin{array}{l}\text { Non-cancer related } \\
\text { mortality (n deaths = 113) }\end{array}$} \\
\hline & HR (95\% CI) & P & HR (95\% CI) & P & HR (95\% CI) & P \\
\hline ASA physical status & $2.2(1.7-2.9)$ & $<0.0001$ & & & $3.1(1.9-5.1)$ & $<0.0001$ \\
\hline $\begin{array}{l}\text { ASA physical status } \\
\text { score IV }\end{array}$ & & & $2.2(1.4-3.5)$ & 0.001 & & \\
\hline $\begin{array}{l}\text { Age } \geq 60 \text { years } \\
\text { Diabetes }\end{array}$ & $2.1(1.4-3.0)$ & $<0.0001$ & $1.8(1.1-2.8)$ & 0.015 & $3.6(1.7-7.4)$ & 0.001 \\
\hline $\begin{array}{l}\text { Histology } \\
\text { Endometrioid } \\
\text { grade 1-2 }\end{array}$ & $1.1(0.82-1.5)$ & 0.551 & - & & $1.5(0.99-2.3)$ & 0.055 \\
Endometrioid & $2.9(2.0-4.1)$ & $<0.0001$ & $3.4(2.2-5.4)$ & $<0.0001$ & $1.2(0.66-2.2)$ & 0.544 \\
\hline
\end{tabular}




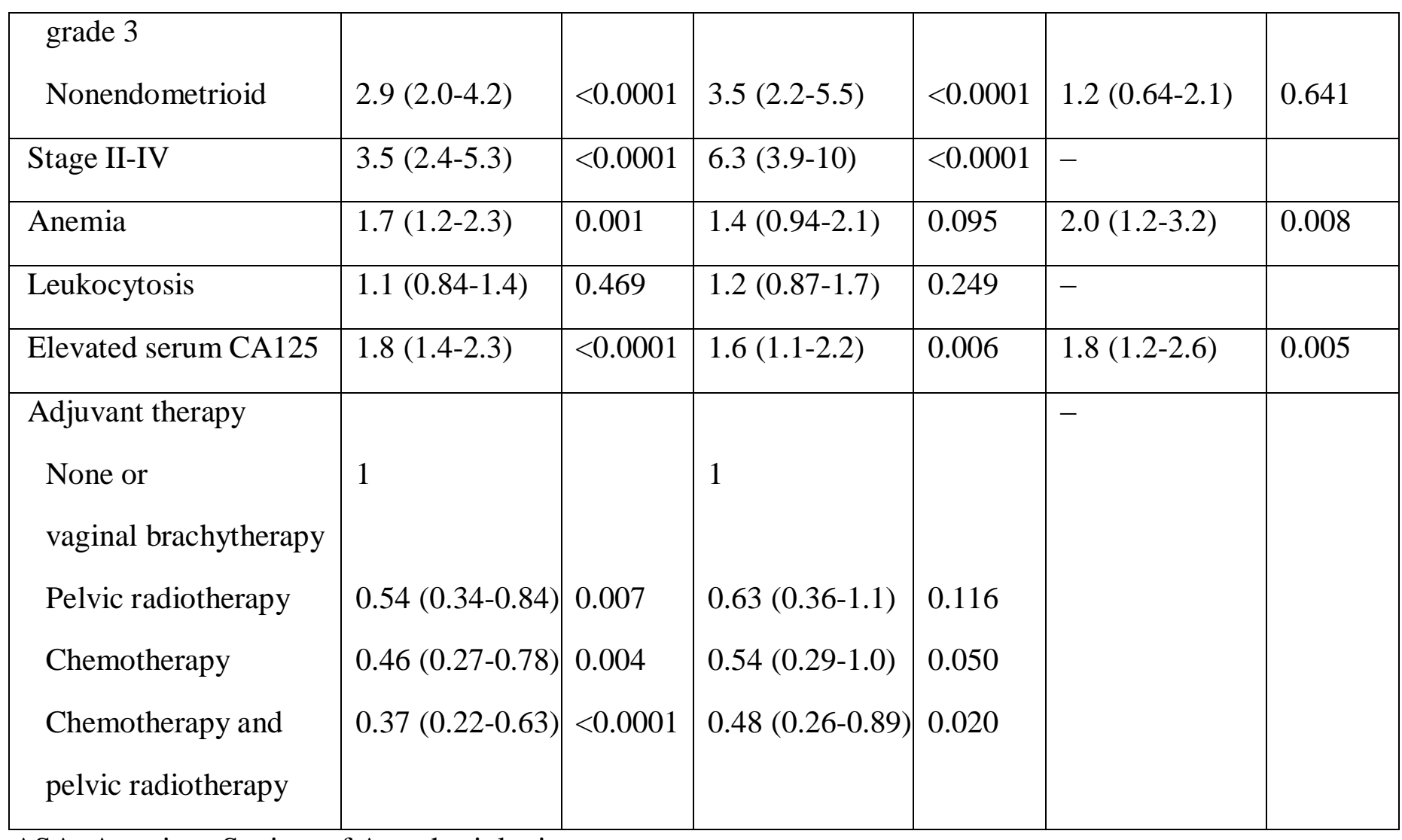

ASA, American Society of Anesthesiologists 

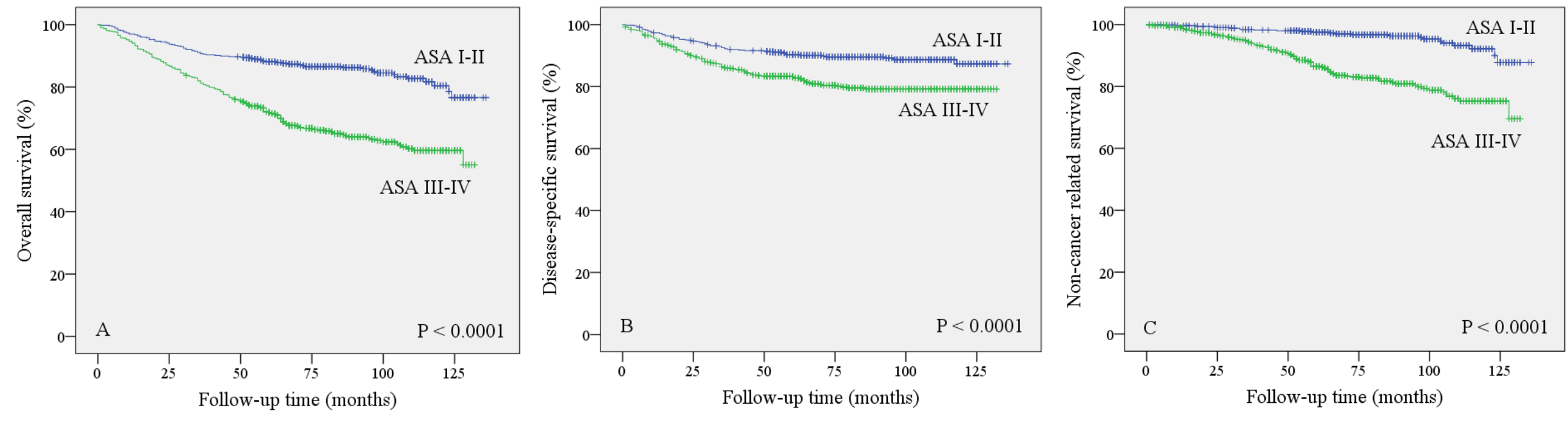

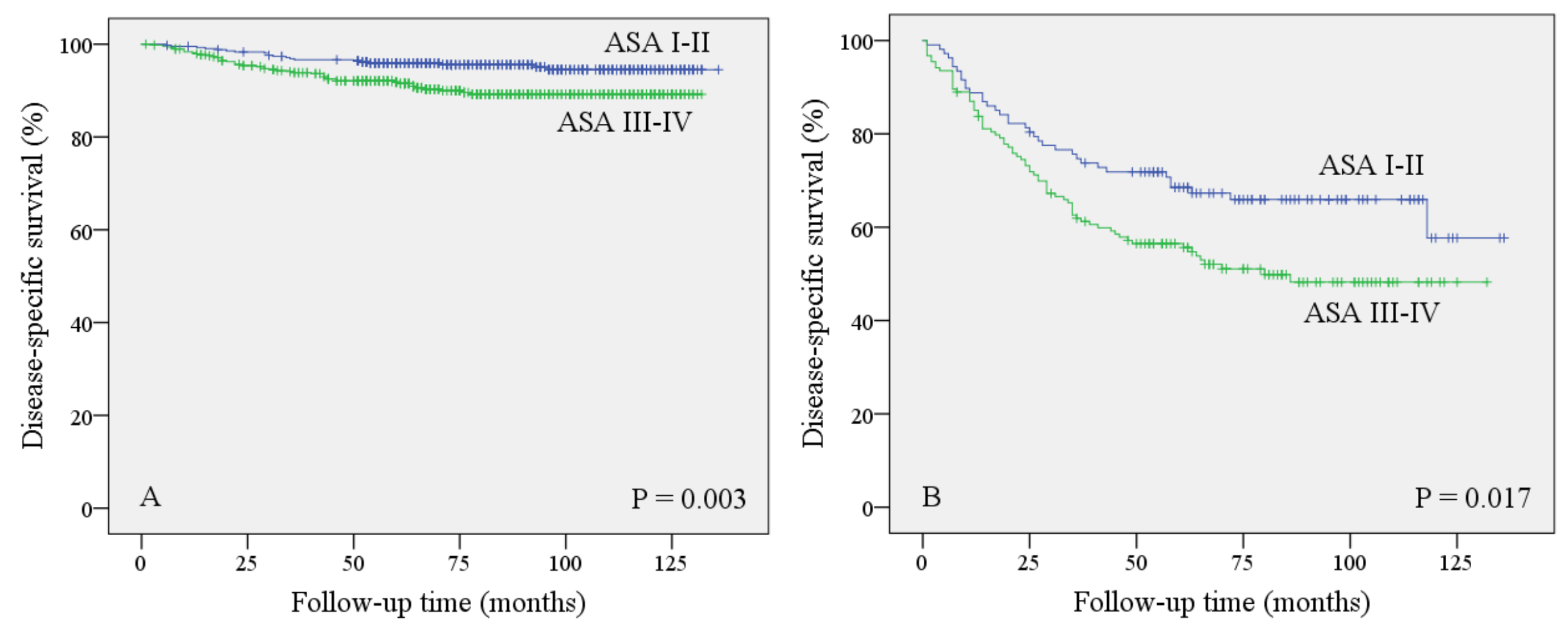